\title{
Differential Inhibition of Macrophage Proliferation by Anti-Transferrin Receptor Antibody ER-MP21: Correlation to Macrophage Differentiation Stage
}

\author{
Pieter J. M. Leenen, ${ }^{*, 1}$ Martin J. Kroos, $†$ Marleen Melis, ${ }^{*}$ Walentina A. T. Slieker, ${ }^{*}$ \\ WILLEM VAN EWIJK,* AND HFNDRIK G. VAN EIJK† \\ *Department of Immunology and †Department of Chemical Pathology, Erasmus University, \\ P.O. Box 1738, 3000 NR Rotterdam, The Netherlands
}

\begin{abstract}
Monoclonal antibodies (mAbs) directed against the transferrin receptor are known to inhibit proliferation of cells due to iron deprivation. Some cell types, however, escape from growth inhibition by a mechanism which is unclear at present. This mechanism is the subject of the present study. We investigated the differential growth inhibition caused by anti-transferrin receptor mAb ER-MP2 1 in connection with the differentiation of murine maerophages $(\mathbf{M} \phi)$. Therefore, we applied two models of $M \phi$ differentiation, namely, culture of bone marrow cells in the presence of M-CSF and a panel of $M \phi$ cell lines ordered in a linear differentiation sequence. In both models we observed that proliferation of $M \phi$ precursors was strongly inhibited by ERMP21. In contrast, proliferation of more mature stages of $\mathbf{M} \phi$ differentiation was hardly affected. Remarkably, iron uptake by $M \phi$ precursor and mature $M \phi$ cell lines was inhibited by ER-MP21 to the same extent. However, mature $M \phi$ cell lines showed an iron uptake twoto threefold higher than that of $M \phi$ precursor cell lines. These observations strongly suggest that mature $\mathbf{M} \phi$ escape from ER-MP21-mediated growth inhibition, because these cells take up more iron than is actually needed for proliferation. Furthermore, we found that enhanced iron uptake by mature $M \phi$ is not necessarily accompanied by a higher cell surface expression of transferrin receptors, thus suggesting an increased recycling of trangferrin receptors in mature $M \phi$. (C) 1990 Academic Press, Inc.
\end{abstract}

\section{INTRODUCIIIUN}

In previous studies we reported on the phenotypical differentiation of murine $\mathbf{M} \phi,{ }^{2}$ using mAbs directed against cell surface molecules $[1,2]$. In search of func-

\footnotetext{
${ }^{1}$ To whom reprint requests should be addressed.

2 Abbreviations used: BSA, bovine serum albumin; M-CSF, macrophage-colony stimulating factor; $\mathrm{M} \phi$, macrophage(s); mAb, monoclonal antibody; SDS, sodium dodecyl sulfate.
}

tionally important epitopes, involved in the regulation of $\mathbf{M} \phi$ differentiation, we produced new mAbs against $\mathrm{M} \phi$ precursor antigens [3] and screened these mAbs for interference with $\mathbf{M} \phi$ colony development in M-CSFstimulated bone marrow cultures. One of these mAbs, termed ER-MP21, appeared to inhibit the in vitro development of $\mathbf{M} \phi$ by hindering $\mathbf{M} \phi$ precursor proliferation. Moreover, the proliferation of $\mathbf{M} \phi$ precursor cell lines was strongly inhibited by ER-MP21. In contrast, mature M $\phi$ cell line proliferation was hardly affected. Pilot studies using ${ }^{59} \mathrm{Fe}$-loaded transferrin indicated that $\mathrm{mAb}$ ER-MP21 inhibited the iron uptake by $\mathbf{M} \phi$ and is therefore most likely directed against the murine transferrin receptor.

Using other anti-transferrin receptor mAbs, a similar differential inhibitory effect on the proliferation of different cell lines has been observed by other investigators as well $[4,5]$. Additionally, different protocols for induction of normal T- and B-lymphocyte proliferation show different degrees of sensitivity toward the inhibitory action of an anti-transferrin receptor $\mathrm{mAb}$ [6]. The mechanism of differential growth inhibition by a particular anti-transferrin receptor mAb remains, however, unclear at present [7]. In general, inhibitory anti-transferrin receptor mAbs cause iron deprivation and thus withhold cells from a vital element in their metabolism $[4,5,8]$. However, it remains to be established whether insensitive cell types are deprived from iron to a lesser extent upon culture with a particular anti-transferrin receptor $\mathrm{mAb}$, or, alternatively, employ additional mechanisms for iron uptake other than the transferrin receptor.

Our initial finding of differential inhibition of $\mathrm{M} \phi$ proliferation by ER-MP21 prompted us to a detailed analysis of the functional properties of this anti-transferrin receptor $\mathrm{mAb}$. In the present study we substantiate the differential inhibition of M $\phi$ proliferation by ER-MP21 and show that growth inhibition is inversely correlated to macrophage maturation. Furthermore, we investigate the mechanism of differential sensitivity for growth in- 
TABLE 1

Cell Lines Used

\begin{tabular}{lll}
\multicolumn{1}{c}{ Cell line } & \multicolumn{1}{c}{ Cell type } & Ref. \\
\hline R2C4 & M $\phi$ precursor hybrid & {$[9]$} \\
R1B1 & M $\phi$ precursor hybrid & {$[9]$} \\
RMB-TG & M $\phi$ precursor, myelocyte & {$[9]$} \\
WEHI-3 & Immature M $\phi$ & {$[10,2]$} \\
RAW309Cr.1 & Mature M $\phi$ & {$[11,2]$} \\
WR19M.1 & Mature M $\phi$ & {$[11,2]$} \\
RLD-1 & Pre-T cell & {$[12,2]$} \\
SP2/0 & B cell & {$[13]$} \\
\hline
\end{tabular}

hibition by ER-MP21, using a panel of $\mathbf{M} \phi$ cell lines arrested in different, previously defined stages of $\mathbf{M} \phi$ differentiation $[2,9]$. The results show that ER-MP21 equally inhibits iron uptake by $\mathbf{M} \phi$ precursor and mature $\mathbf{M} \phi$ cell lines. However, mature $\mathbf{M} \phi$ cell lines exhibit a level of iron uptake significantly higher than that of $\mathrm{M} \phi$ precursor cell lines. These data suggest that mature M $\phi$ escape from ER-MP21-mediated growth inhibition because these cells take up iron in amounts greater than those needed merely for proliferation.

\section{MATERIALS AND METHODS}

Mice. Male and female BALB/c mice were purchased from Olac (Bicester, Oxon, UK). Animals were kept under clean, routine laboratory conditions with free access to food and water.

Cells and culture conditions. The cell types and differentiation stages of the cell lines used in the present study are summarized in Table 1. The M $\phi$ precursor cell lines $\mathrm{R} 1 \mathrm{~B} 1$ and $\mathrm{R} 2 \mathrm{C} 4$ were hybrids obtained by fusion of RMB-TG myeloid cells and $M \phi$ derived from MCSF-stimulated bone marrow culture [9]. All cell lines were cultured in DMEM (Flow Laboratories, Irvine, Scotland), supplemented with $10 \%$ SERUM-PLUS (KC Biological, I.enexa, KS) and antihiotics. MCSF-stimulated bone marrow cultures were performed in 24-wells culture plates (Costar, Cambridge, MA) with L-cell-conditioned medium as the source of M-CSF, essentially as described by Van der Meer $e t$ al. [14].

Quantification of cell proliferation. Cell lines were set into culture in 96-wells culture plates in low density, ensuring exponential growth after 3 days. Then, after 3 days of culture in the presence or absence of mAbs, resulting cell numbers were quantified using the colorimetric MTT assay as described by Mosmann [15]. For M-CSF-stimulated bone marrow cultures, resulting progeny was quantified similarly after 7 days.

Antisera and conjugates. The IgG2a anti-transferrin receptor $\mathrm{mAb}$ ER-MP21, as well as the control IgG2a mAbs ER-MP20, ER-MP39, and ER-MP42, were produced by hybridomas, obtained after fusion of Y3 myeloma cells with spleen cells from a Lewis rat that had been immunized with $\mathrm{M} \phi$ precursor hybrid cells [3]. Hybridoma cells, secreting the anti-transferrin receptor mAb H129.121 (Ref. [16]) were kindly provided by Dr. Michel Pierres (INSERM-CNRS, Marseille, France). For application in culture, $\mathrm{mAbs}$ were purified from ascitic fluid (1/1 diluted in PBS) by $18 \% \mathrm{Na}_{2} \mathrm{SO}_{4}$ precipitation (w/v; final concentration), desalted using Sephadex G-25M columns (Pharmacia, Uppsala, Sweden), and filter-sterilized. In flow-cytometric experiments, we used FI'C-coupled $F(a b)_{2}$ fragments of rabbit anti-(ratIgG) (Cappel, Malvern, PA) to detect rat mAb binding to cells. $m A b$ and transferrin purification and radiolabeling. ER-MP21 $\mathrm{mAb}$ purification for binding affinity studies was achieved by specific adsorption and subsequent elution from a column of mouse anti-rat kappa (MARK-1) coupled Sepharose-4B beads [17]. Murine transferrin was purified from pooled normal mouse serum by affinity chromatography over rabbit anti-rat transferrin coupled to Sepharose-4B (Ref. [18]), taking advantage of immunological cross-reactivity between rat and mouse transferrin. Iron removal from purified transferrin and reloading with ${ }^{69} \mathrm{Fe}$ was performed as described before, using the nitrilotriacetic acid method [19]. Radio-iodination of ER-MP21 $\mathrm{mAb}$ and transferrin was performed with ${ }^{125} \mathrm{I} \mathrm{Na}$ (Amersham Radiochemical Centre, UK) using lodogen reagent (Pierce Chemical, Rockford, IL) as catalyst [20].

${ }^{59} \mathrm{Fe}$ uptake studies. Cellular iron uptake from ${ }^{59} \mathrm{Fe}$-loaded transferrin was determined essentially as described by Verhoef and Noordeloos [21]. Briefly, cells were harvested, washed at least twice with serum-free DMEM, and resuspended in DMEM supplemented with $0.5 \%(\mathrm{w} / \mathrm{v})$ bovine serum albumin. Cells $\left(1-4.10^{6} / \mathrm{ml}\right)$ were incubated in siliconized vials for $30 \mathrm{~min}$ at $37^{\circ} \mathrm{C}$ in the presence or absence of $\mathrm{mAb}(20 \mu \mathrm{g} / \mathrm{ml})$. Then, ${ }^{59} \mathrm{Fe}$-loaded transferrin $\left(5 \mu \mathrm{g} / 10^{6}\right.$ cells $)$ was added. At various times, duplicate samples of $0.5 \mathrm{ml}$ were taken and iron uptake was stopped immediately by adding $5 \mathrm{ml}$ ice-cold PBS. Cells were washed three times in ice-cold PBS and cell-bound radioactivity was measured in a Packard Autogamma 500-C spectrometer. In all experiments, nonspecific cell-bound radioactivity-usually less than $1 \mathrm{pmol} / 10^{6}$ cells-was subtracted from the obtained experimental values.

Radiolabeled $\mathrm{mAb}$ and $T$ f binding studies. For determination of binding of ${ }^{125}$ I-labeled ER-MP21 or ${ }^{126}$ I-labeled transferrin, all steps were performed on ice. Cells were washed with DMEM and resuspended at a concentration of $5.10^{6}$ cells $/ 200 \mu \mathrm{l}$ in DMEM containing $0.5 \% \mathrm{BSA}$ as described above. In competitive binding studies, preincubation was performed with $100 \mu$ l potentially blocking nonradioactive reagents for $30 \mathrm{~min}$. Next, $100 \mu \mathrm{l}{ }^{125}$ I-labeled ER-MP21 or ${ }^{125}$ I-labeled transferrin, diluted in DMEM + 0.5\% BSA to the concentration indicated, was added to the cells. In binding affinity determination, dilution series were used with and without 100 -fold excess of unlabeled ligand to assess nonspecific binding. Cells were incubated under regular mixing for $3 \mathrm{~h}$ to equilibrate ligand binding. Then, cell-bound and free radioactivities from $100 \mu \mathrm{l}$ aliquots were separated by spinning the cells through $300 \mu \mathrm{l}$ of an oil mixture of dinonylphtalate and dibutylphtalate (3:7.5) in 0.4-ml reaction vessels (Eppendorf, Hamburg, FRG), as adapted from Klausner et al. [22]. The tubes were frozen in liquid nitrogen and cut with a heated surgical blade. Cell-bound and free radioactivity were measured as indicated. For affinity determination, binding data were analyzed according to Scatchard [23].

Antigen characterization. The molecular mass of the antigens recognized by ER-MP21 and H129.121 mAb was determined by surface * iodination of cells, followed by specific immunoprecipitation and SDS-PAGE analysis, essentially as described by Pont et al. [24].

Immunofluorescence labeling and flow-cytometric unalysis. Cells were labeled for immunofluorescence as described previously [2]. Analysis was performed on a FACScan (Becton-Dickinson, Sunnyvale, CA) with logarithmic amplification. Antibody binding was quantified by interpolation on a calibration scale, using quantitative fluorescent micro bead standards (Becton-Dickinson). Thus, antibody binding is expressed as soluble FITC molecular equivalents (mol.eqFITC).

\section{RESULTS}

\section{ER-MP21 Recognizes the Murine Transferrin Receptor but Does Not Compete with Transferrin Binding}

In order to discover epitopes that are of functional importance in the course of $M \phi$ differentiation, we 


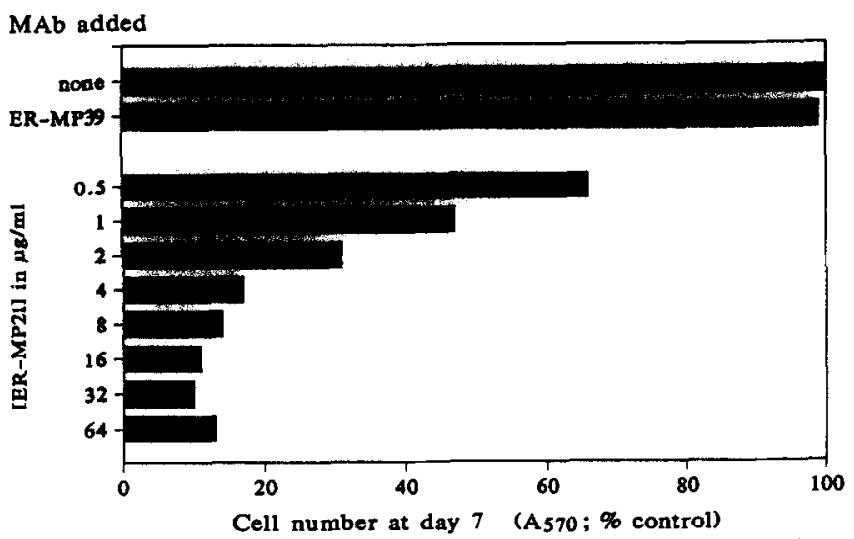

FIG. 1. Inhibition of macrophage development in M-CSF stimulated bone marrow culture by ER-MP21. Murine bone marrow was cultured with M-CSF in the presence or absence of different concentrations of $\mathrm{mAb}$. After 7 days the mature $\mathbf{M} \phi$ progeny obtained was quantified using the colorimetric MTT assay.

screened several anti-M $\phi$ precursor mAbs for interference with $\mathrm{M} \phi$ differentiation in M-CSF stimulated bone marrow cultures. The rat IgG2a mAb, termed ER-MP21, appeared to inhibit the development of mature bone marrow-derived $\mathbf{M} \phi$ in a dose-dependent manner (Fig. 1). Maximal inhibition was reached at ER-MP21 concentrations higher than $5 \mu \mathrm{g} / \mathrm{ml}$. In contrast, control rat IgG2a mAbs (ER-MP39 shown as example), which recognize other molecules on $\mathbf{M} \phi$ precursor cells, caused no inhibition of $\mathbf{M} \phi$ development, even at the highest concentration tested $(30 \mu \mathrm{g} / \mathrm{ml})$.

Inhibition of iron uptake by anti-transferrin receptor $\mathrm{mAbs}$ is a well-known cause of inhibition of proliferation [7]. Therefore, we examined the effect of ER-MP21 on cellular iron uptake. Figure 2 shows that ER-MP21 indeed inhibits iron uptake from ${ }^{59} \mathrm{Fe}$-loaded transferrin in a dose-dependent manner. The question arises whether

\section{TABLE 2}

ER-MP21 and Transferrin Have Different Binding Sites on Transferrin Receptors

\begin{tabular}{llcc}
\hline & & \multicolumn{2}{c}{$\begin{array}{c}\text { Ligand bound } \\
\text { (pmole/3 } \times 10^{6} \text { cells) }\end{array}$} \\
\cline { 3 - 4 } Preincubation & ${ }^{125}$ I-labeled ligand & Expt. I & Expt. II \\
\hline - & Mu-Tf & 0.62 & 0.52 \\
Rat-Tf $^{b}$ & Mu-Tf & 0.48 & nd \\
NMS $^{c}$ & Mu-Tf & nd & 0.30 \\
ER-MP21 $^{b}$ & Mu-Tf & 0.63 & 0.45 \\
ER-MP20 $^{b}$ & Mu-Tf & 0.60 & 0.43 \\
- & ER-MP21 & 0.45 & 0.44 \\
Rat-Tf $^{e}$ & ER-MP21 & 0.41 & nd \\
NMS $^{c}$ & ER-MP21 & nd & 0.45 \\
ER-MP21 $^{e}$ & ER-MP21 & 0.07 & 0.08 \\
ER-MP20 $^{e}$ & ER-MP21 & 0.42 & 0.40 \\
\hline
\end{tabular}

Note. RAW309Cr.1 cells were assayed for competitive binding of murine transferrin (Mu-Tf), anti-transferrin receptor mAb ERMP21, rat transferrin (rat-Tf), or normal mouse serum (NMS) on ice, using the concentrations indicated. Rat mAb ER-MP20 served as irrelevant IgG2a control.

${ }^{a}$ Ligand $\left[{ }^{125} \mathrm{I}-\mathrm{Mu}-\mathrm{Tf}\right]=1 \times 10^{-6} \mathrm{M}$.

${ }^{b}$ Preincubation [rat-Tf] $=[$ ER-MP21] $=[$ ER-MP20] $=1.3$ $\times 10^{-5} M$.

' Preincubation NMS $=33 \%$.

${ }^{d}$ Ligand $\left[{ }^{125}\right.$ I-ER-MP21] $=1 \times 10^{-7} \mathrm{M}$.

${ }^{e}$ Preincubation [rat-Tf] $=[$ ER-MP21] $=[$ ER-MP20] $=1.3$ $\times 10^{-6} M$.

inhibition of iron uptake is mediated through competition between transferrin and ER-MP21 for the same binding site at the transferrin receptor. To investigate this possibility, RAW309Cr.1 cells were incubated with ${ }^{125}$ I-labeled transferrin or ${ }^{125}$ I-labeled ER-MP21, with or without preincubation with excess unlabeled transferrin, ER-MP21, or irrelevant IgG2a mAb ER-MP20. The results, presented in Table 2, clearly show that ER-
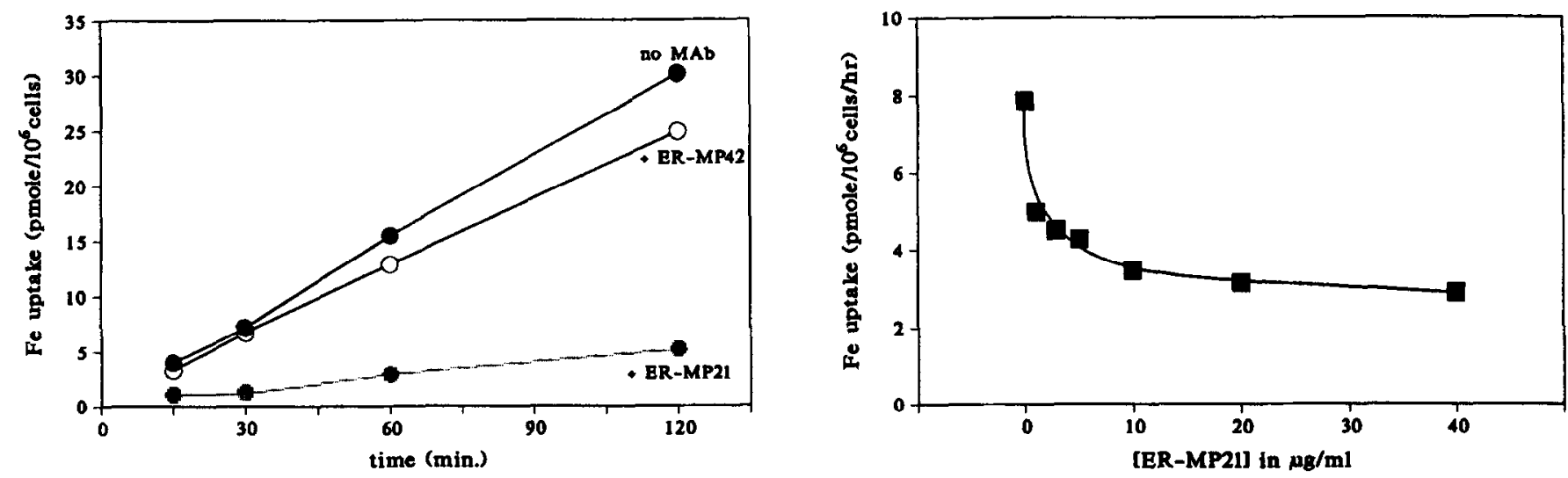

FIG. 2. Inhibition of iron uptake by ER-MP21. (left) Specific ${ }^{59} \mathrm{Fe}$-uptake by the mature M $\phi$ cell line WR19M.1 in the absence and the presence of $25 \mu \mathrm{g} / \mathrm{ml}$ ER-MP21 or $25 \mu \mathrm{g} / \mathrm{ml}$ ER-MP42 control mAb. The slight inhibition of iron uptake in the presence of ER-MP42 was not observed in other experiments. (right) Dose-dependency of ER-MP21-mediated inhibition of ${ }^{50} \mathrm{Fe}$-uptake by the mature $\mathrm{M} \phi$ cell line RAW309Cr.1. Cells were incubated with $\mathrm{mAb}$ for $30 \mathrm{~min}$ at $37^{\circ} \mathrm{C}$ prior to the addition of ${ }^{50} \mathrm{Fe}$-transferrin. 


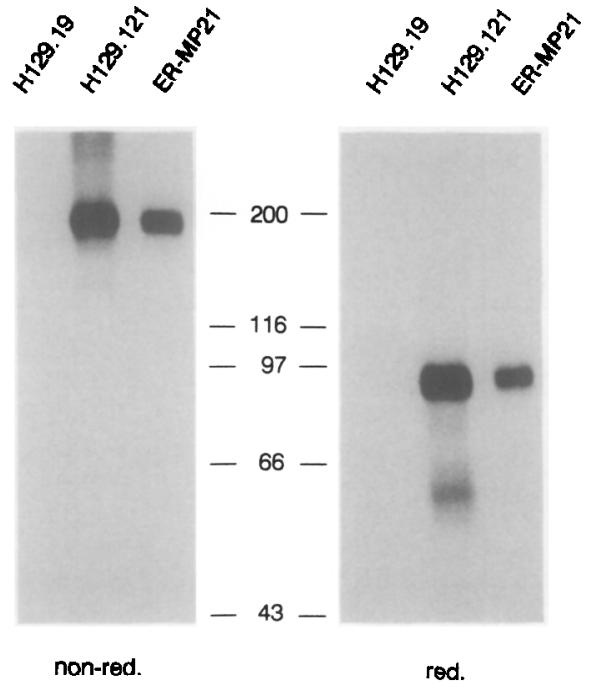

FIG. 3. Immunoprecipitation of transferrin receptors by mAbs ER-MP21 and H129.121 from ${ }^{125}$ I-labeled WR19M.1 cells. Precipitated transferrin receptors were run on a $10 \%$ polyacrylamide gel under reducing and nonreducing conditions. mAb H129.19, directed against murine $\mathrm{CD} 4$, was used to detect nonspecifically binding proteins.

MP21 and transferrin have different binding sites on the transferrin receptor, since preincubation of cells with ER-MP21 caused no significant inhibition of ${ }^{125}$ I-labeled transferrin binding, and vice versa. Similar results were obtained when R2C4 M $\phi$ precursor cells were used (data not shown). We have no reasonable explanation for the relatively high nonspecific binding of ${ }^{125}$ I-labeled transferrin in these experiments.

To confirm the notion that ER-MP21 recognizes the murine transferrin receptor, we immunoprecipitated the ER-MP21 antigen from ${ }^{125}$ I-labeled cells and assessed its molecular mass by SDS-PAGE followed by autoradiography. Figure 3 shows that ER-MP21 precipitates an antigen of the same apparent molecular mass as the murine transferrin receptor, precipitated by $\mathrm{mAb} \mathrm{H} 129.121$ (Ref. [16]). Both mAbs recognize a complex of $200 \mathrm{kDa}$, consisting of two identical chains of $100 \mathrm{kDa}$.

In summary, rat mAb ER-MP21 inhibits murine $\mathbf{M} \phi$ development in M-CSF-stimulated bone marrow culture. ER-MP21 recognizes the murine transferrin receptor, since the mAb inhibits cellular uptake of ${ }^{59} \mathrm{Fe}$-transferrin and precipitates a glycoprotein of molecular mass identical to that of the murine transferrin receptor. However, ER-MP21 does not interfere with transferrin binding to the receptor.

\section{ER-MP21 Inhibits Proliferation of M $\phi$ Precursors, but Not of Mature M $\phi$}

We then approached the question whether proliferation of different $\mathbf{M} \phi$ differentiation stages is equally affected by ER-MP21. This was investigated in two in vitro models of $\mathbf{M} \phi$ differentiation, viz., $\mathrm{M}$-CSF-stimulated bone marrow culture and a panel of $\mathbf{M} \phi$ cell lines, which previously has been ordered in a differentiation sequence on the basis of functional and phenotypical characteristics [2].

In M-CSF-stimulated bone marrow cultures, $\mathrm{M} \phi$ develop from $\mathbf{M} \phi$ precursors by the action of the lineagespecific growth factor M-CSF. The relative maturity of the proliferating cells steadily increases with time during the first days of culture [14]. In order to assess the inhibitory effect of ER-MP21 on proliferation of cells in different stages of $\mathbf{M} \phi$ differentiation, we added $\mathrm{mAb}$ ( 30 $\mu \mathrm{g} / \mathrm{ml}$ ) to bone marrow cultures at different days and quantified the resulting $\mathbf{M} \phi$ progeny at Day 7 after onset of culture. Parallel cultures were irradiated on the same days with 1500 rad $\gamma$-radiation as $100 \%$ growth inhibition controls. This radiation dose prevented further proliferation of the cells, but allowed terminal differentiation. When ER-MP21 was added at Day 0, the total cell count at Day 7 was less than $10 \%$ of the untreated controls (Fig. 4, left). In contrast, addition of $\mathrm{mAb}$ at Day 4 of culture hardly affected the magnitude of resulting progeny at Day 7 (93\% of control). For all irradiated and ER-MP21-treated cultures, the number of cell cycles was calculated that treated cultures were left behind compared to untreated controls. The retarded cell cycle numbers thus obtained for the irradiated cultures were taken as $100 \%$ growth inhibition controls. Then, the retarded cell cycle numbers obtained for ER-MP21treated cultures were expressed relative to the irradiation-treated controls, thus revealing a relative growth inhibition for the $\mathbf{M} \phi$ precursors present on the respective days of $\mathrm{mAb}$ addition (Fig. 4, right). Clearly, bone marrow $\mathrm{M} \phi$ precursors (assayed at Day 0 ) were very sensitive to growth inhibition by ER-MP21, whereas the more mature cells present in culture at Day 4 were hardly affected.

The second model of $\mathrm{M} \phi$ differentiation, in which the inhibitory effect of ER-MP21 was examined, was a panel of $\mathrm{M} \phi$ cell lines aligned according to their differentiation stage [2], supplemented with the $\mathbf{M} \phi$ precursor hybrids R2C4 and R1B1 (Ref. [9]; see Table 1). Figure 5 shows that proliferation of $\mathrm{M} \phi$ precursor $(\mathrm{R} 2 \mathrm{C} 4, \mathrm{R} 1 \mathrm{~B} 1$, and RMB-TG) and immature M $\phi$ (WEHI-3) cell lines was strongly inhibited. Most $\mathrm{R} 2 \mathrm{C} 4$ cells died in the presence of ER-MP21. In contrast, proliferation of the mature M $\phi$ cell lines RAW309Cr.1 and WR19M.1 was only marginally influenced by ER-MP21. A similar differential growth inhibition was observed for non-M $\phi$ cell lines as well: RLD-1 pre-T cells died during 3 days of culture in the presence of ER-MP21, whereas proliferation of SP2/ 0 myeloma cells was not inhibited.

Thus, in two models of $\mathbf{M} \phi$ differentiation we observed that anti-transferrin receptor mAb ER-MP21 strongly inhibited proliferation of $\mathbf{M} \phi$ precursors, whereas prolif- 

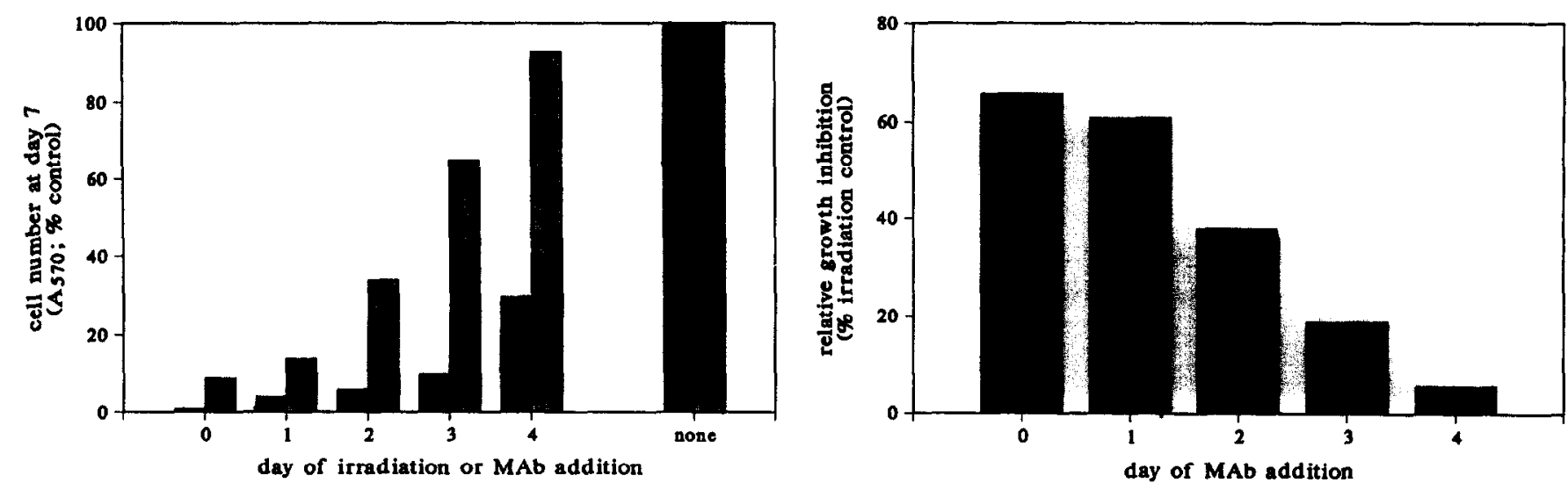

FIG. 4. Differentiation stage-dependent inhibition of M $\phi$ proliferation by ER-MP21 in M-CSF-stimulated bone marrow cultures. (left) $M \phi$ progeny at Day 7 in cultures, treated in parallel on various days by irradiation (1500 rad ${ }^{~}{ }^{0} \mathrm{Co} \gamma$-radiation, black bars) or addition of ERMP21 (30 $\mu \mathrm{g} / \mathrm{ml}$ final concentration, hatched bars). Cell numbers were quantified using the MTT assay and expressed relative to untreated controls. (right) Relative growth inhibition calculated from data represented in Fig. 4, left. For mAb- or radiation-treated cultures, the number of cell cycles needed to reach the cell number in untreated controls was calculated. Values obtained for irradiated cultures were taken as $100 \%$ inhibition controls and values for ER-MP21-treated cultures were expressed relative to these.

eration of more mature stages of $\mathbf{M} \phi$ differentiation was hardly affected.

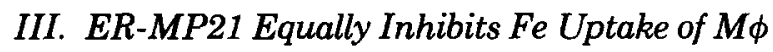 \\ Precursor and Mature $M \phi$ Cell Lines, but the Latter Show a Higher Fe Uptake}

We then approached the question of what the mechanism of differentiation-stage correlated inhibition of $\mathbf{M} \phi$ proliferation by ER-MP21 was. Therefore, we investigated the iron uptake by the various cell lines in the absence and the presence of ER-MP21 (Fig. 6, left). The presence of ER-MP21 $(20 \mu \mathrm{g} / \mathrm{ml})$ reduced the uptake of ${ }^{59} \mathrm{Fe}$ for all tested cell lines to an average of $35 \%$ (range

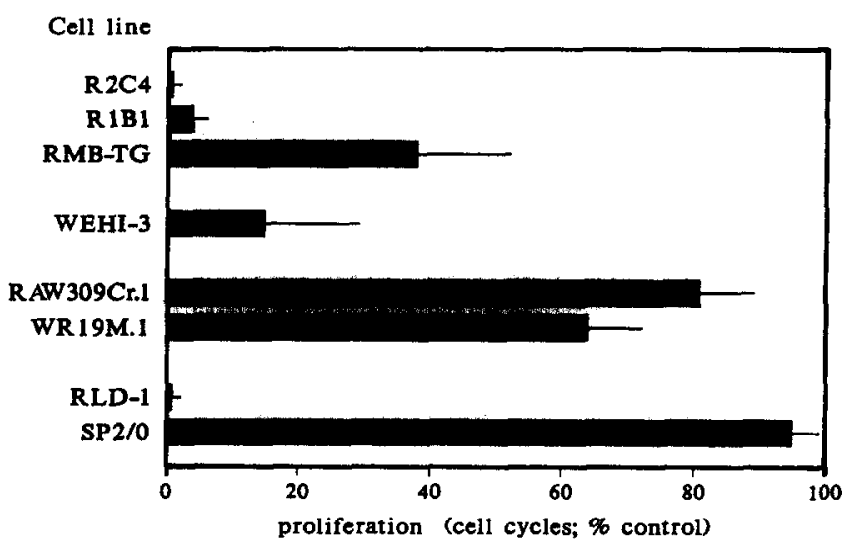

FIG. 5. Differentiation stage-dependent inhibition of $\mathbf{M} \phi$ proliferation by ER-MP21 in a panel of $\mathbf{M} \phi$ cell lines. $\mathbf{M} \phi$ precursor (R2C4, R1B1, RMB-TG), immature M $\phi$ (WEHI-3), mature $\mathrm{M} \phi$ (RAW309Cr.1, WR19M.1), pre-T (RLD-1), and myeloma (SP2/0) cell lines were cultured in the presence or the absence of ER-MP21 $(20 \mu \mathrm{g} /$ $\mathrm{ml}$ ) or control IgG2a mAb (not shown). After 3 days, cell numbers in parallel cultures were quantified using the MTT assay. Proliferation is given as number of cell cycles performed relative to untreated controls.
28-51\%) of untreated control value, irrespective of differentiation stage or cell type. However, mature $\mathbf{M} \phi$ cell lines showed a ${ }^{59} \mathrm{Fe}$ uptake two- to threefold higher than that of $\mathbf{M} \phi$ precursor and immature $\mathbf{M} \phi$ cell lines, both in the absence and in the presence of ER-MP21. This difference cannot be ascribed to significant differences in cell size of the various cell lines as determined by flow-cytometric forward light scatter analysis (data not shown). Hence, for the cell lines of the $M \phi$ lineage, the proliferation in the presence of ER-MP21 correlates highly to the residual iron uptake in the presence of the $\mathrm{mAb}$ (Fig. 6, right; $r=0.86$ ). This relationship appears to be strongly cell-type dependent. SP2/0 myeloma cells, namely, grew undisturbedly on a level of residual iron uptake as low as $1 \mathrm{pmol} / 10^{6}$ cells $/ \mathrm{h}$, whereas proliferation of $\mathrm{R} 2 \mathrm{C} 4 \mathrm{M} \phi$ precursor cells was completely inhibited at the same low level of iron uptake.

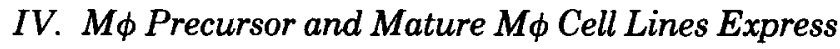 Similar Numbers of Slightly Different Transferrin Receptors}

The most likely explanation for increased iron uptake by mature $\mathbf{M} \phi$ cell lines, compared to that of $\mathbf{M} \phi$ precursor and immature $\mathbf{M} \phi$ cell lines, is an increased cell surface expression of transferrin receptors. To affirm this supposition, we examined the transferrin receptor expression by the $\mathbf{M} \phi$ cell line panel semiquantitatively using the flow cytometer. Simultaneously, ${ }^{59} \mathrm{Fe}$ uptake was determined for another part of the same cell preparation. In different experiments, transferrin receptor expression on $\mathbf{M} \phi$ precursor and immature $\mathbf{M} \phi$ cell lines varied from approximately 1 to $4 \times 10^{5}$ mol.eq. FITC, depending on cell line and culture density (Fig. 7). Indeed, the mature M $\phi$ cell line WR19M.1 showed an increased transferrin receptor expression $\left(5-6 \times 10^{5}\right.$ 

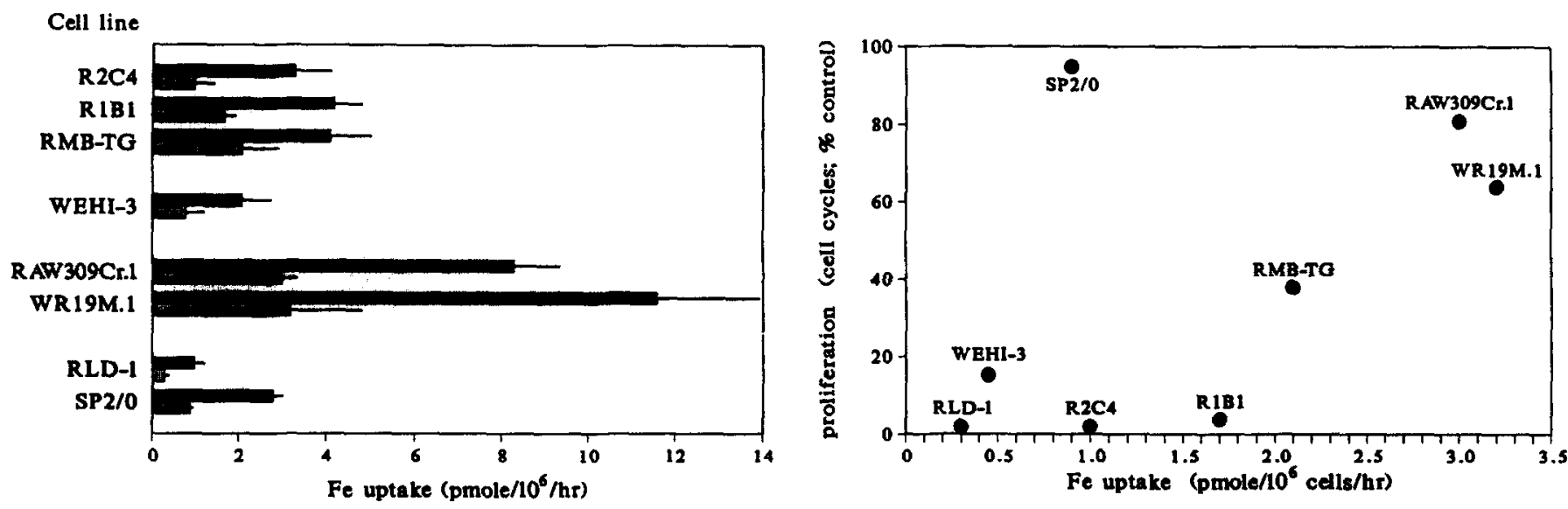

FIG. 6. (left) ER-MP21-mediated inhibition of iron uptake by $\mathrm{M} \phi$ and non-M $\phi$ cell lines. ${ }^{59} \mathrm{Fe}$ uptake was measured in $\mathrm{M} \phi$ precursor (R2C4, R1B1, RMB-TG), immature M $\phi$ (WEHI-3), mature M $\phi$ (RAW309Cr.1, WR19M.1), pre-T (RLD-1), and myeloma (SP2/0) cell lines in the absence (black bars) or presence (hatched bars) of ER-MP21. Data represent the mean of at least four determinations per cell line \pm SEM. (right) Correlation between residual iron uptake by the various cell lines and proliferation in the presence of ER-MP21. Data represented in Figs. 5 and 6, left, are combined in this figure.

mol.eq. FITC), which related to an increased iron uptake. Surprisingly, the mature RAW309Cr.1 cells showed a transferrin receptor expression comparable to the expression found for $\mathbf{M} \phi$ precursor and immature $\mathrm{M} \phi$ cell lines $\left(2 \times 10^{5}\right.$ mol.eq. FITC). Iron uptake by RAW309Cr.1 cells, however, was three-fold higher. Apparently, RAW309Cr.1 cells endocytosed as much as three times the amount of transferrin-bound iron than did $\mathbf{M} \phi$ precursor cells, using the same number of cell surface transferrin receptors.

As such, the expression of transferrin receptors by the investigated $\mathrm{M} \phi$ cell lines showed no obvious correlation with the expression of other differentiation stage-related markers (cf. Refs. [2, 9]). The observation that

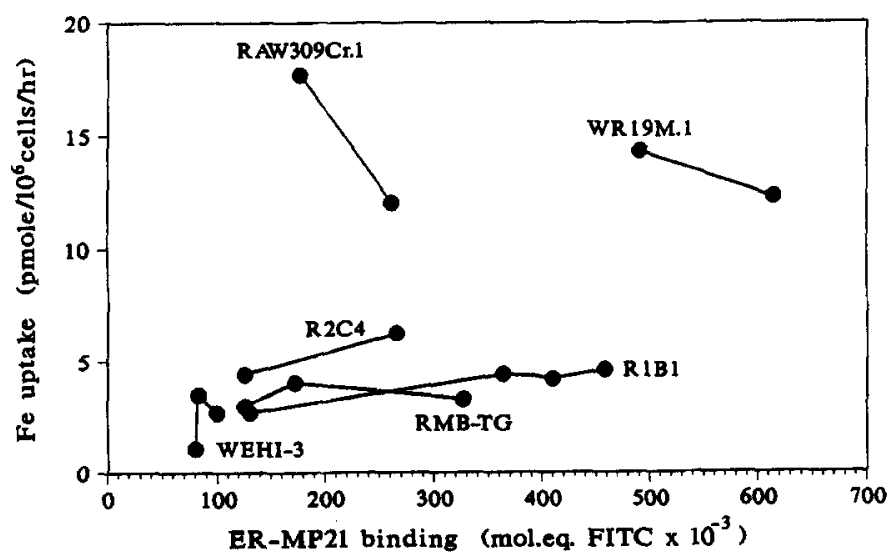

FIG. 7. Relationship between cell surface transferrin receptor expression and iron uptake for different $\mathbf{M} \phi$ cell lines. Part of a cell preparation was processed for immunofluorescence and used to quantify ER-MP21 binding by flow cytometry. For another part of the same preparation ${ }^{58} \mathrm{Fe}$ uptake was measured. Each point represents the ERMP21 binding and ${ }^{59} \mathrm{Fe}$ uptake determined in a single experiment. Data from different experiments on the same cell line are connected.
RAW309Cr.1 cells internalized far more iron than $\mathbf{M} \phi$ precursor cells, using the same number of transferrin receptors, raised the question whether transferrin receptors on mature $\mathrm{M} \phi$ cell lines, in particular on RAW309Cr.1 cells, are structurally different from transferrin receptors on $\mathbf{M} \phi$ precursor and immature $\mathbf{M} \phi$ cell lines. From binding affinity studies using ${ }^{125}$ T-labeled ER-MP21 we obtained circumstantial evidence that this is indeed the case. Figure 8 (left) shows that ERMP21 bound to R2C4 transferrin receptors with a single affinity $\left(K_{d}=19-23 \times 10^{-9} M\right)$. Remarkably, RAW309Cr.1 cells showed two types of receptors with different affinities for ER-MP21 (Fig. 8, middle). Highaffinity receptors on RAW309Cr.1 cells bound the mAb more avidly $\left(K_{d}=7.3 \times 10^{-9} \mathrm{M}\right.$; range 5.7-9.0 $\left.\times 10^{-9} \mathrm{M}\right)$ compared to $\mathrm{R} 2 \mathrm{C} 4$ transferrin receptors. These analyses also confirmed the preceding data (Fig. 7), namely, that R2C4 and RAW309Cr.1 cells express similar numbers of transferrin receptors. In different experiments ERMP21 binding sites on R2C4 cells ranged from 2-13 $\times 10^{4}$ per cell (Fig. 8 , left), whereas the high affinity binding sites on RAW309Cr.1 cells ranged from 3-10 $\times 10^{4}$ per cell (Fig. 8, middle), depending on culture density of the cells [25].

A likely candidate for the ER-MP21 low-affinity receptor on RAW309Cr. 1 cells $\left(K_{d} \approx 4.5 \times 10^{-7} M\right)$ is the receptor for the Fc part of IgG, which is known to be expressed by mature $M \phi$ cell lines [26]. Therefore, we performed the same binding affinity studies in the presence of $1.3 \times 10^{-6} M$ isotype control mAb ER-MP20. This concentration is about equimolar to the highest concentration ${ }^{125}$ I-ER-MP21 used $\left(1.10^{-6} M\right)$. Clearly, the binding pattern of ER-MP21, revealing two affinities, was not significantly altered by the addition of irrelevant $\mathrm{mAb}$ (Fig. 8, right). It is therefore unlikely that the 

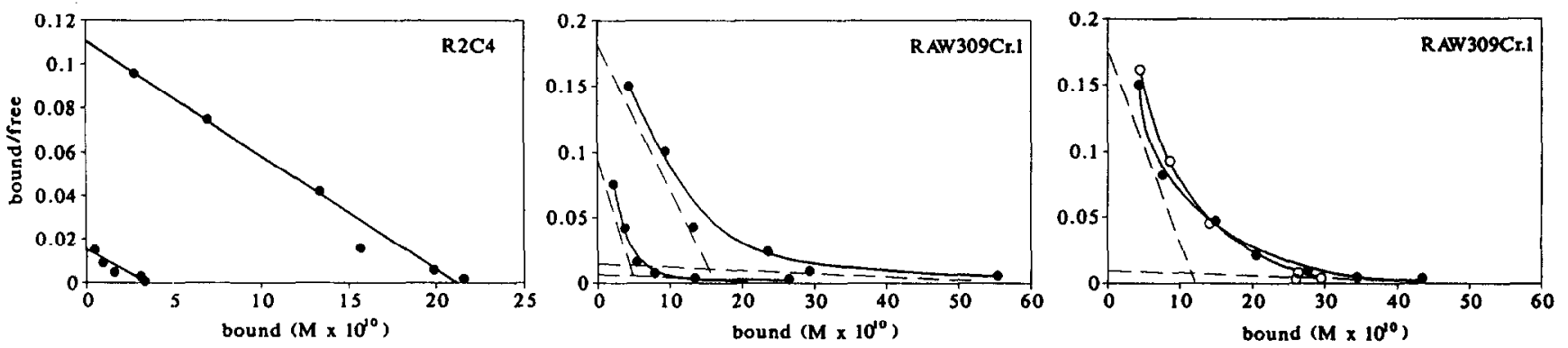

FIG. 8. (left) Scatchard analysis of ER-MP21 binding to R2C4 M $\phi$ precursor cells. Results from two representative experiments, revealing different numbers of binding sites, but similar affinities, are given. (middle) Scatchard analysis of ER-MP21 binding to RAW309Cr.1 mature M $\phi$ cells in two different experiments. (right) Scatchard analysis of ER-MP21 binding to RAW309Cr.1 cells in the presence (O) and absence (•) of irrelevant rat IgG2a mAb ER-MP20.

low-affinity ER-MP21 receptor on RAW309Cr.1 cells is identical to the Fc receptor.

Thus, the different binding affinities of ER-MP21 to transferrin receptors on RAW309Cr.1 and R2C4 cells suggest that transferrin receptors on mature and immature $\mathbf{M} \phi$ are structurally different.

\section{DISCUSSION}

In search of mAbs directed against functionally important epitopes on $\mathbf{M} \phi$ precursor cells, we selected IgG2a mAb ER-MP21 for its inhibitory capacity of M $\phi$ development in M-CSF-stimulated bone marrow cultures. ER-MP21 appeared to be directed against the murine transferrin receptor, since this $\mathrm{mAb}$ inhibited uptake of transferrin-bound ${ }^{59} \mathrm{Fe}$ and precipitated, as the anti-transferrin receptor $\mathrm{mAb} \mathrm{H} 129.121$, a homodimer of $200 \mathrm{kDa}$ [16]. Like other IgG anti-transferrin receptor mAbs $[6,7,16,27]$, ER-MP21 bound to an epitope different from the transferrin-binding site.

In general, growth inhibition by anti-transferrin receptor mAbs is caused by iron starvation of cells $[4,5$, 8]. The underlying mechanism, proposed for IgG antitransferrin receptor $\mathrm{mAbs}$, is that $\mathrm{mAb}$ binding decreases transferrin receptor recycling between the cell surface and intracellular compartments $[8,28,29]$. Furthermore, transferrin receptor degradation is enhanced, possibly by inappropriate intracellular routing of the complex consisting of transferrin, transferrin receptor, and $\mathrm{mAb}[5,29]$. Both decreased recycling and enhanced degradation cause a reduced cell surface expression of transferrin receptors, leading to reduced iron uptake with concomitant growth inhibition in the presence of anti-transferrin receptor $\mathrm{mAb}$.

For $\mathrm{M} \phi$ precursors, we indeed observed a strong inhibition of proliferation by anti-transferrin receptor $\mathrm{mAb}$ ER-MP21. Most likely, this inhibition is mediated by the mechanism described above. However, proliferation of more mature stages of $\mathrm{M} \phi$ differentiation was hardly affected by ER-MP21. We observed this differentiation stage-related inhibition of $\mathbf{M} \phi$ proliferation in two inde- pendent models of $\mathbf{M} \phi$ differentiation, namely, M-CSFstimulated bone marrow culture and a panel of $\mathbf{M} \phi$ cell lines, aligned in differentiation sequence. Thus, with increasing maturity, proliferating $\mathbf{M} \phi$ become refractory to inhibition by anti-transferrin receptor mAb. The highly sensitive $M \phi$ precursor cells are found around the CFU-GM/CFU-M stage of differentiation: CFU-M are the main M-CSF responsive cells in the bone marrow, and these were shown to be strongly inhibited (Fig. 4, left). Furthermore, the equally sensitive $\mathbf{M} \phi$ precursor cell lines appear to be arrested around the CFU-GM stage of differentiation [2]. More mature, insensitive stages of differentiation, occurring at Day 4 of bone marrow culture and represented by the mature $\mathrm{M} \phi$ cell lines in the panel, are around the monoblast/monocyte stage $[2,14]$.

A comparable lack of growth inhibition by an antitransferrin receptor $\mathrm{mAb}$, inhibitory for other cell types, has been reported previously for murine $L$ cells [4] as well as for different lymphocyte subsets [6]. The mechanism of differential growth inhibition, however, remained largely obscure [7]. Our cell line model of $\mathbf{M} \phi$ differentiation offered a unique opportunity to study this mechanism, since large numbers of cells with different sensitivities are available from a single hemopoietic lineage. In ${ }^{59} \mathrm{Fe}$-uptake studies, we observed that ER-MP21 equally inhibited iron uptake by sensitive and insensitive $\mathrm{M} \phi$ cell lines. Thus, the mechanism by which ERMP21 blocks iron uptake is most likely similar for sensitive and insensitive cell lines. However, insensitive mature $\mathrm{M} \phi$ cell lines showed an iron uptake both in the presence and in the absence of ER-MP21 two to three times higher than that of immature $\mathbf{M} \phi$ cell lines. Apparently, the diminished iron uptake by mature $\mathbf{M} \phi$ cell lines, in the presence of the $\mathrm{mAb}$, is still sufficient for undisturbed proliferation. In contrast, reduction of the lower level of iron uptake in $\mathbf{M} \phi$ precursor cell lines caused by the action of the mAb, leads to severe inhibition of proliferation or even cell death. This indicates that $\mathrm{M} \phi$ precursor cell lines apply virtually all endocytosed iron for growth and proliferation, whereas mature 
M $\phi$ cell lines normally take up more iron than needed merely for growth. A similar explanation is, most likely, valid for the differential growth inhibition of bone marrow-derived $\mathbf{M} \phi$ : insensitive monoblasts/monocytes at Day 4 of culture may take up a multiple of the amount of iron taken up by CFU-M.

How do mature $\mathbf{M} \phi$ take up more iron than $\mathbf{M} \phi$ precursors? The most likely mechanism is the expression of an increased number of transferrin receptors [30]. In this study, we indeed observed for the mature WR19M.1 cells a transferrin receptor expression higher than that of the investigated $\mathrm{M} \phi$ precursor cell lines. This might serve as an explanation for increased iron uptake by WR19M.1 cells. However, RAW309Cr.1 cells showed a two to three times increased iron uptake, compared to that of $\mathbf{M} \phi$ precursor cell lines, and yet expressed similar numbers of transferrin receptor on the cell surface. Thus, increased iron uptake is not necessarily accompanied by increased transferrin receptor expression. Our binding affinity analyses of ER-MP21 revealed that RAW309Cr.1 transferrin receptors are presumably structurally different from transferrin receptors on $\mathbf{M} \phi$ precursors. ER-MP21 bound with single affinity to transferrin receptors on $\mathrm{R} 2 \mathrm{C} 4$ cells, whereas two affinities were found for receptors on RAW309Cr.1 cells. The high affinity binding - to the transferrin receptor-was more avid, compared to the binding to $\mathrm{R} 2 \mathrm{C} 4$ cells, thus indicating a structural difference between the binding sites on both cell types. The low-affinity ER-MP21 receptor on RAW309Cr.1 cells appeared to be distinct from the receptor for the Fc part of IgG. Unfortunately, we have no further clues to its nature and relationship to the transferrin receptor. Interestingly in this context, Andreesen et al. [30] showed that mature human $\mathrm{M} \phi$ transferrin receptors bind their natural ligand transferrin with different affinity compared to that of other cell types, thus supporting the view that mature $\mathbf{M} \phi$ express structurally different transferrin receptors. The present results suggest that structurally different transferrin receptors on mature $\mathbf{M} \phi$ account for a more efficient endocytosis of transferrin-bound iron. Increased efficiency in this respect is presumably mediated by an increased receptor recycling of transferrin receptors between cell membrane and intracellular compartments. It is noteworthy that a similar increase in transferrin receptor recycling appears to occur during erythroid differentiation [31].

An alternative explanation for the increased iron uptake by mature $M \phi-$ using the same number of transferrin receptors-might be the existence of additional pathways of iron uptake, independent from the transferrin receptor. For example, receptors for glycan moieties on transferrin, such as the asialoglycoprotein receptor or the mannose receptor, might mediate endocytosis of transferrin [32]. Moreover, fluid-phase endocytosis would be a highly feasible mechanism of alternative iron uptake for the actively endocytosing mature $\mathbf{M} \phi$ [33]. Such alternative mechanisms would not be blocked by anti-transferrin receptor mAb ER-MP21. To investigate this possibility, we studied whether the competitive protein asialofetuin or the competitive saccharides mannan and $N$-acetyl-glucosamine affected ${ }^{59} \mathrm{Fe}$ uptake by mature $\mathrm{M} \phi$ cell lines or caused increased inhibition of ${ }^{59} \mathrm{Fe}$ uptake in the presence of ER-MP21 (unpublished results). Unequivocally, these agents had no effects on cellular iron uptake. Furthermore, the level of fluidphase endocytosis in mature $\mathbf{M} \phi$, measured as uptake of ${ }^{59} \mathrm{Fe}$-citrate, could by no means account for a two to three times increased iron uptake, compared to that of $\mathbf{M} \phi$ precursor cells (data not shown). These alternative pathways of iron uptake must be excluded therefore as mechanisms of enhanced iron uptake by mature $\mathbf{M} \phi$. Moreover, the similar relative inhibition of iron uptake by ER-MP21 for M $\phi$ precursor and mature $\mathrm{M} \phi$ cell lines (Fig. 6, left) indicates that the same mechanism, i.e., transferrin receptor-mediated iron uptake, is active in both cell types.

Why do mature $\mathbf{M} \phi$ endocytose as much as two to three times the amount of iron needed for proliferation? Iron is incorporated in a number of vital proteins, such as heme-containing globins, cytochromes, and iron-containing enzymes. Furthermore, iron is stored intracellularly in ferritin $[34,35] . M \phi$ are known to be an important link in the iron metabolism by the phagocytosis of senescent erythrocytes $[36,37]$. In connection with this, $\mathrm{M} \phi$ constitute a considerable iron storage in the body. The development of this function is reflected by the increasing intracellular ferritin content during terminal differentiation of $\mathrm{M} \phi$ [38]. Both the present results and those obtained by Andreesen et al. [30, 38] suggest that mature $\mathrm{M} \phi$ can accumulate iron for storage purposes, not only by phagocytosis of erythrocytes, but also by means of transferrin receptor-mediated iron uptake.

This work was supported by a research grant (80-25) from the Netherlands Asthma Foundation. We thank Mr. Tar van Os for preparation of the figures and Mrs. Cary Meijerink-Clerkx for perfect secretarial assistance.

\section{REFERENCES}

1. Leenen, P. J. M., Willmer, U., Falkenberg, F. W., Jansen, A. M. A. C., and Van Ewijk, W. (1986) in Leukocytes and Host Defense (Oppenheim, J. J., and Jacobs, D. M., Eds), pp. 289-294, Liss, New York.

2. Leenen, P. J. M., Jansen, A. M. A. C., and Van Ewijk, W. (1986) Differentiation 32, 157-164.

3. Leenen, P. J. M., Melis, M., and Van Ewijk, W. (1990) Eur. J. Immunol. 20, 27-34.

4. Trowbridge, I. S., Lesley, J., and Schulte, R. (1982) J. Cell. Physiol. 112, 403-410.

5. Lesley, J., and Schulte, R. (1985) Mol. Cell. Biol. 5, 1814-1821.

6. Kemp, J. D., Thorson, J. A., McAlmont, T. H., Horowitz, M., Cowdery, J. S., and Ballas, Z. K. (1987) J. Immunol. 138, 24222426. 
7. Trowbridge, I. S., and Shackelford, D. A. (1986) Biochem. Soc. Symp. 51, 117-129.

8. Taetle, R., Castagnola, J., and Mendelsohn, J. (1986) Cancer Res. 46, 1759-1763.

9. Leenen, P. J. M., Slieker, W. A. T., Melis, M., and Van Ewijk, W. (1990) Eur. J. Immunol. 20, 15-25.

10. Warner, N. L., Moore, M. A. S., and Metcalf, D. (1969) J. Natl. Canc. Inst. 43, 963-977.

11. Raschke, W. C., Baird, S., Ralph, P., and Nakoinz, I. (1978) Cell 15, 261-267.

12. De Both, N. J., Rhijnsburger, E. H., and Van Ewijk, W. (1983) Int. J. Cancer 32, 501-506.

13. Shulman, M., Wilde, C. D., and Köhler, G. (1978) Nature (London) 276, 269-270.

14. Van der Meer, J. W. M., Van de Gevel, J. S., and Van Furth, R. (1983) J. Reticuloendothel. Soc. 34, 203-225.

15. Mosmann, T. (1983) J. Immunol. Methods 65, 55-63.

16. Van Agthoven, A., Goridis, C., Naquet, P., Pierres, A., and Pierres, M. (1984) Eur. J. Biochem. 140, 433-440.

17. Bazin, H., Xhurdebise, L.-M., Burtonboy, G., Lebacq, A.-M., De Clery, L., and Cormont, F. (1984) J. Immunwl. Methods 66, 261-269.

18. Van Eijk, H. G., and Van Noort, W. L. (1976) J. Clin. Chem. Clin. Biochem. 14, 475-478.

19. Van der Heul, C., De Jeu-Jaspars, C. M. H., Kroos, M. J., and Van Eijk, H. G. (1978) Biochim. Biophys. Acta 51 1, 430-441.

20. Markwell, M. A. K., and Fox, C. F. (1978) Biochemistry 17, 4807-4817.

21. Verhoef, N. J., and Noordeloos, P. J. (1977) Clin. Sci. Mol. Med. 52, 87-96.
22. Klausner, R. D., Van Renswoude, J., Ashwell, G., Kempf, C., Schechter, A. N., Dean, A., and Bridges, K. R. (1983) J. Biol. Chem. 258, 4715-4724.

23. Scatchard, G. (1949) Ann. N. Y. Acad. Sci. 51, 660-672.

24. Pont, S., Regnier-Vigouroux, A., Naquet, P., Blanc, D., Pierres, A., Marchetto, S., and Pierres, M. (1985) Eur. J. Immunol. 15, 1222-1228.

25. Musgrove, E., Rugg, C., Taylor, I., and Hedley, D. (1984) J. Cell. Physiol. 118, 6-12.

26. Morahan, P. S. (1980) J. Reticuloendothel. Soc. 27, 223-245.

27. Takei, F. (1983) J. Immunol. 130, 2794-2797.

28. Schmidt, J. A., Marshall, J., Hayman, M. J., Ponka, P., and Beug, H. (1986) Cell 46, 41-51.

29. Lesley, J., Schulte, R., and Woods, J. (1989) Exp. Cell Res. 182, 215-233.

30. Andreesen, R., Sephton, R. G., Gadd, S., Atkins, R. C., and De Abrew, S. (1988) Blut 57, 77-83.

31. Mulford, C. A., and Lodish, H. F. (1988) J. Biol. Chem. 263, 5455-5461.

32. Van Berkel, T. J. C., Dekker, C. J., Kruijt, J. K., and Van Eijk, H. G. (1987) Biochem. J. 243, 715-722.

33. Steinman, R. M., and Cohn, Z. A. (1972) J. Cell Biol. 55, 186204.

34. Aisen, P., and Listowsky, I. (1980) Annu. Rev. Biochem. 49, 357393.

35. Jacobs, A. (1985) Curr. Top. Hematol. 5, 25-62.

36. Finch, C. A., and Huebers, H. (1982) N. Engl. J. Med. 306, 15201528.

37. Deiss, A. (1983) Semin. Hematol. 20, 81-90.

38. Andreesen, R., Osterholz, J., Bodemann, H., Bross, K. J., Costabel, U., and Löhr, G. W. (1984) Blut 49, 195-202. 